\title{
Multiwavelength All-Optical Clock Recovery
}

\author{
C. Johnson, K. Demarest, C. Allen, R. Hui, Senior Member, IEEE, K. V. Peddanarappagari, and B. Zhu
}

\begin{abstract}
Multiwavelength clock recovery is especially desirable in systems that use wavelength-division-multiplexed technology. A multiwavelength clock-recovery device can greatly simplify costs by eliminating the need to have a separate regenerator for each wavelength. This letter discusses multiwavelength all-optical clock recovery using stimulated Brillouin scattering.
\end{abstract}

Index Terms - Clock recovery, optical fiber communication, optical fiber device, optical nonlinearity, wavelength-division multiplexing.

\section{INTRODUCTION}

$\mathbf{F}$ UTURE broad-band integrated services digital networks (B-ISDN) may operate at terabit per second (Tb/s) data rates using time-division multiplexing (TDM) and/or wavelength-division multiplexing (WDM). These networks will likely use all-optical 3-R regenerators, which reamplify, reshape, and retime the incoming data. The latter two of these operations would require all-optical clock-recovery devices. Although multiwavelength amplification is already a reality with erbium-doped fiber amplifiers (EDFA's), multiwavelength clock-recovery devices have not yet been demonstrated in the literature.

Previously reported all-optical clock-recovery devices include mode-locked fiber ring lasers [1], mode-locked laser diodes [2], self-pulsating laser diodes [3], and optical tanks [4]. Most of these devices require prior knowledge of the bitrate. An exception is a Brillouin optical active filter, which is able to operate without a prior knowledge of the bitrate [5]. To our knowledge, there have been no reports of multiwavelength clock recovery.

In this letter, we present results showing dual-wavelength all-optical clock recovery using a Brillouin active filter. The remainder of this letter is divided into three sections. In Section II, we cover the theory of the device; in Section III, we discuss the results; and in Section IV, we summarize the advantages of this device.

\section{THEORY}

Our device can be classified as a Brillouin active filter, which relies on the process of stimulated Brillouin scattering (SBS). In SBS, a strong pump wave causes molecular vibrations, which result in a change in the index of refraction. Thus, an acoustic wave (Bragg grating) is formed. Part of a pump wave will scatter off of the Bragg grating, resulting

Manuscript received February 22, 1999; revised March 29, 1999.

The authors are with the Information and Telecommunication Technology Center, University of Kansas, Lawrence, KS 66044 USA.

Publisher Item Identifier S 1041-1135(99)05164-2.

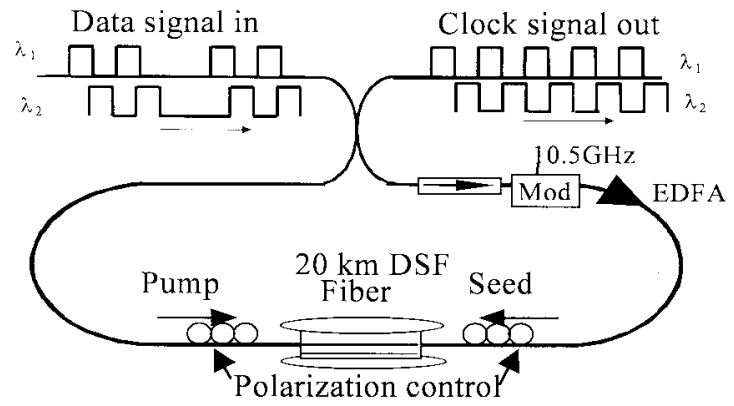

Fig. 1. Block diagram of the Brillouin active-filter clock-recovery device.

in a downshifted wave (called a Stokes wave) traveling in the opposite direction from the pump and the acoustic waves. The Stokes wave will experience exponential amplification if the pump wave is above a threshold that is dependent on the characteristics of the fiber. If an input signal is injected at the Stokes wavelength, SBS can be used for amplification of that signal. The SBS-induced amplification has a narrow-gain bandwidth.

This narrow bandwidth, nonlinear amplification process can be exploited in a system to recover the clock of an incoming return-to-zero (RZ) optical data stream by selectively amplifying the strongest clock tones in the data spectrum, which are shifted from the carrier by the bitrate and multiples of the bitrate. This filtering effect is caused by the strongest tones generating the most backscattered energy in an SBS process, thereby providing greater gain for tones of a signal at the Stokes wavelength. The RZ clock output is obtained by effectively dumping some of the energy from the bit slots that contain ones into the bit slots that contain zeros. In this way, the Brillouin active filter converts an RZ data stream with random ones and zeros into an RZ clock.

There have been a variety of Brillouin optical active filters reported in the literature [5], [6]. Our device, shown in Fig. 1, is an extension of these previously reported Brillouin active filters. Here, an input optical RZ data stream is injected into a directional coupler, producing counter-rotating waves in a loop that contains $20 \mathrm{~km}$ of dispersion-shifted fiber (DSF). The polarization controllers maintain the same polarization state for both the pump and Stokes waves. The clockwise propagating signal is downshifted by the Brillouin shift of the DSF fiber $(\approx 10.5 \mathrm{GHz})$ using a Mach-Zehnder modulator (MZM), biased to obtain double-sideband, suppressed-carrier modulation. The downshifted seed wave (Stokes wave) is amplified by the counterpropagating pump wave SBS, since the counterpropagating waves inside the DSF fiber are separated in frequency by exactly the Brillouin shift. However, 


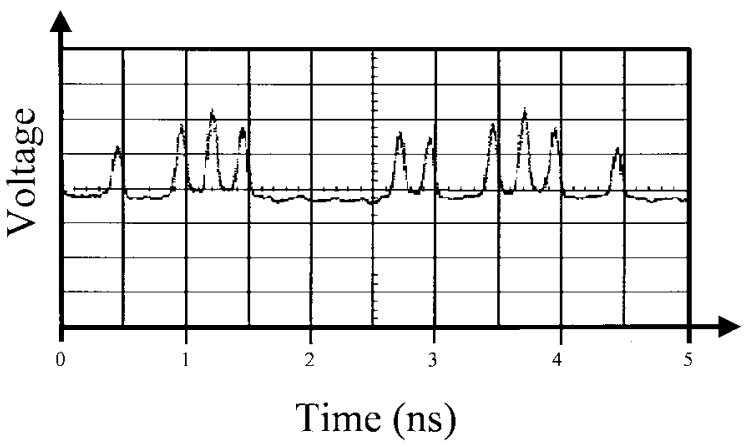

Fig. 2. $10-\mathrm{Gb} / \mathrm{s}\left(2^{7}-1\right)$ pseudorandom optical input $(500 \mathrm{ps} / \mathrm{div})$.

only the clock-related continuous-wave $(\mathrm{CW})$ tones in the seed waveform have sufficient power to exceed the Brillouin threshold, so the data portion of the seed spectrum is attenuated relative to the clock. Therefore, this device operates as an active, optical filter. Since the same data stream is used for the pump and the Stokes seed, this device is bit-rate independent.

Multiwavelength clock recovery is possible from this device for the following reasons. First, the Brillouin gain bandwidth of the DSF fiber $(\approx 20 \mathrm{MHz})$ is much narrower than the spectra of the data signals, thus allowing each seed signal to interact only with its own unshifted pump signal. This attribute is common to all single wavelength Brillouin active filters, but also makes multiwavelength clock recovery possible if each channel's clock tone power levels are all above the Brillouin threshold. Second, the erbium-doped fiber amplifier (EDFA) in the loop of our device provides the gain necessary for multiwavelength operation for moderate input powers; i.e., less than $15.5 \mathrm{dBm} /$ channel. Although the Brillouin shift varies inversely with the pump wavelength, multiwavelength clock recovery is possible for signals within a 3-nm bandwidth for the DSF fiber used in our device.

The data rates of each wavelength need not be the same. The only cost of adding each additional wavelength channel is that the input power to the Brillouin active filter or the gain of the EDFA in the loop must be increased by $3 \mathrm{~dB}$ for each additional wavelength.

\section{RESULTS}

Fig. 2 shows a representative input waveform for either of the two channels. The input RZ pulses had pulsewidths of $24 \mathrm{ps}$ and repetition rates of $10 \mathrm{GHz}$. The input data stream was encoded with a $2^{7}-1$ pseudorandom bit sequence generated by an HP70843A bit-error-rate tester (BERT). The data streams were then amplified to an average power of $35 \mathrm{~mW}$ per channel using an EDFA and then injected into the input port of the directional coupler. The coupling ratio of the directional coupler was $90: 10$. The clockwise Stokes signal was generated using a dual-electrode MZM, which was biased to produce a double-sideband suppressed-carrier output spectrum. The downshifted Stokes spectrum was then amplified by a low-noise figure $(N F=5 \mathrm{~dB})$ EDFA with output power of $4 \mathrm{dBm}$ per channel and then sent through

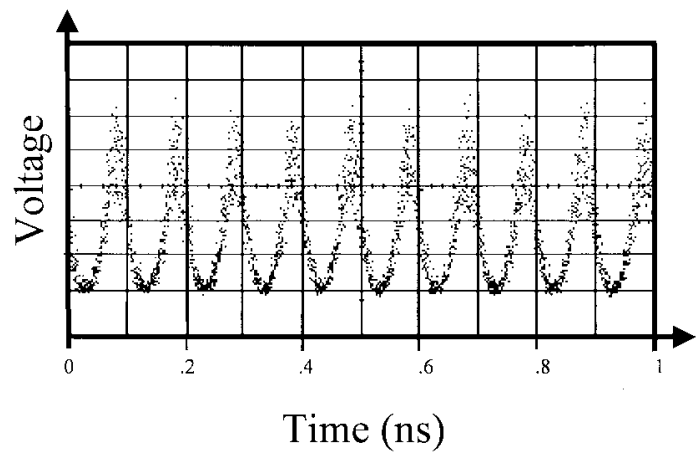

Fig. 3. Recovered optical clock at $1557.4 \mathrm{~nm}$ (100 ps/div).

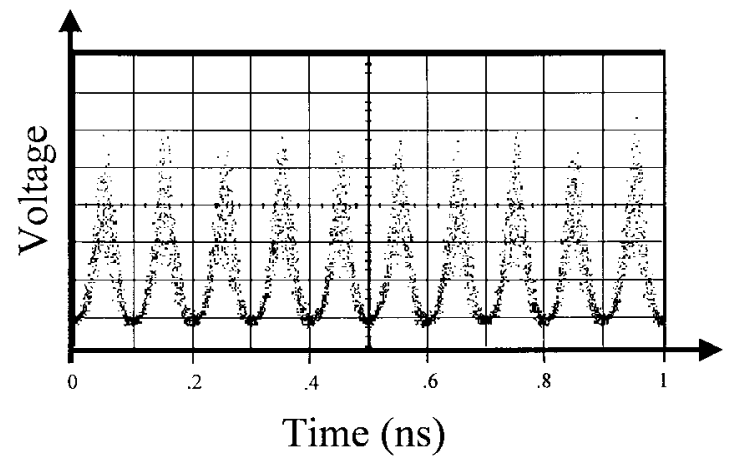

Fig. 4. Recovered optical clock at $1556.5 \mathrm{~nm}$ (100 ps/div).

the dispersion-shifted fiber to act as a seed for the SBS process.

The output waveforms for the 1557.4 and $1556.5 \mathrm{~nm}$ channels are shown in Figs. 3 and 4, respectively. The relative jitter of the 1557.4- and 1556.5-nm clocks were 1.2 and 1.3 ps, respectively, where relative jitter is defined by $\sigma_{\mathrm{RMS}}=$ $\sqrt{\sigma_{\mathrm{CLOCK}}^{2}-\sigma_{\mathrm{DATA}}^{2}}$. These waveforms clearly show that the device is capable of simultaneously extracting the clocks from both data streams.

\section{CONCLUSION}

In this letter, we demonstrated a clock-recovery technique employing an active filter using stimulated Brillouin scattering. The WDM compatibility of this clock-recovery technique makes it attractive for future optical networks. By using a single device to recover the clocks of a number of optical inputs, this technology could be integrated into future WDM networks, thereby eliminating the need for costly regenerators for each wavelength.

Although the total wavelength span of our clock-recovery device was roughly $3 \mathrm{~nm}$, this span could be increased by either of two methods. The first would be to use a fiber loop with a larger Brillouin-gain bandwidth, which could be accomplished by introducing strain into the fiber [7]. The second would be to replace the single modulator in the loop with multiple modulators and corresponding wavelength filters that provide different modulation shifts for different wavelength bands. Using either of these techniques, wide-band multiwavelength clock recovery may be possible. 


\section{REFERENCES}

[1] A. Ellis, K. Smith, and D. Patrick, "All optical clock recovery at bit rates up to $40 \mathrm{Gbit/s,"} \mathrm{Electron.} \mathrm{Lett.,} \mathrm{vol.} \mathrm{29,} \mathrm{pp.} \mathrm{1741-1743,}$ 1993.

[2] R. Ludwig, A. Ehrhardt, W. Pieper, E. John, N. Agrawal, H.-J. Ehrke, L. Küller, and H. Weber, "40 Gbit/s demultiplexing experiment with $10 \mathrm{GHz}$ all-optical clock recovery using a modelocked semiconductor laser," Electron. Lett., vol. 32, no. 4, pp. 327-329, 1996.

[3] P. Barnsley, H. Wickes, G. Wickens, and D. Spirit, "All-optical clock recovery from $5 \mathrm{Gbit} / \mathrm{s} \mathrm{RZ}$ data using a self-pulsating $1.56 \mu \mathrm{m}$ laser diode," IEEE Photon. Technol. Lett., vol. 3, pp. 942-945, Oct. 1991.
[4] M. Jinno and T. Matsumoto, "Optical tank circuits used for all-optical timing recovery," IEEE J. Quantum Electron., vol. 28, pp. 897-900, Apr. 1992.

[5] D. Butler, L. Douglas, J. Wey, M. Chbat, G. Burdge, and J. Goldhar, "Optical clock recovery from a data stream of an arbitrary bit rate by use of stimulated Brillouin scattering," Opt. Lett., vol. 20, no. 6, pp. $560-562,1995$.

[6] H. Kawakami, T. Mitamoto, T. Kataoka, and K. Hagimoto, "All-optical timing clock extraction using multiple wavelength pumped Brillouin amplifier," IEICE Trans. Commun., vol. E78-B, no. 5, pp. 694-701, 1995.

[7] T.-O. Tsun, A. Wada, T. Sakai, and R. Yamauchi, "Novel method of using white spectral probe signals to measure Brillouin gain spectra of pure silica core fibers," Electron. Lett., vol. 28, no. 3, pp. 247-249, Jan. 1992. 